\title{
Neonatal bilirubin production-conjugation imbalance: effect of glucose-6-phosphate dehydrogenase deficiency and borderline prematurity
}

\author{
M Kaplan, M Muraca*, H J Vreman, C Hammerman, M T Vilei, F F Rubaltelli, D K Stevenson
}

Arch Dis Child Fetal Neonatal Ed 2005;90:F123-F127. doi: 10.1136/adc.2004.058313

See end of article for authors' affiliations auth affiliations

Correspondence to: Dr Kaplan, Department of Neonatology, Shaare Zedek Medical Center, PO Box 3235, Jerusalem 91031, Israel; kaplan@ cc.huji.ac.il

Accepted 7 October 2004

\begin{abstract}
Objective: To evaluate relations between production and conjugation of bilirubin in the pathophysiology of jaundice in glucose-6-phosophate dehydrogenase (G6PD) deficient neonates.

Methods: Term and borderline premature (35-37 weeks gestational age), healthy, male, G6PD deficient neonates were studied close to the beginning of the 3rd day. Blood carboxyhaemogobin corrected for inspired $\mathrm{CO}$ ( $\mathrm{COHbc}$; an index of bilirubin production) and serum total conjugated bilirubin (TCB; a reflection of bilirubin conjugation) were measured in simultaneously drawn blood samples by gas chromatography and reverse phase high performance liquid chromatography respectively. A bilirubin production-conjugation index comprising $\mathrm{COHbc/TCB}$ was determined; a high index reflects imbalance between the bilirubin production and conjugation processes. $\mathrm{COHbc}$ and $\mathrm{TCB}$ individually and the production-conjugation index were studied in relation to serum total bilirubin (STB) concentration.

Results: Fifty one G6PD deficient neonates were sampled at 51 (8) hours. $\mathrm{COHbc}$ values did not correlate with STB $(r=0.22, p=0.15)$. TCB did correlate inversely with STB $(r=-0.42, p=0.004)$, and there was a positive correlation between the production-conjugation index and STB $(r=0.45, p=0.002)$. The production-conjugation index (median (interquartile range)) was higher in the premature $(\mathrm{n}=8)$ than term neonates $(2.31(2.12-3.08) \vee 1.05(0.53-1.81), p=0.003)$. This difference was the result of changes in TCB.

Conclusions: The data show that jaundice in G6PD deficient neonates is the result of an imbalance between production and conjugation of bilirubin with a tendency for inefficient bilirubin conjugation over increased haemolysis in its pathogenesis. Borderline premature infants are at especial risk of bilirubin production-conjugation imbalance.
\end{abstract}

a low index. Conversely, high bilirubin production relative to conjugation should result in a raised index.

Two risk factors highly associated with neonatal hyperbilirubinaemia with the potential of bilirubin encephalopathy or kernicterus include G6PD deficiency and borderline prematurity (35-37 weeks gestation). The former is a common $\mathrm{X}$ linked enzymatic deficiency, which may nowadays be encountered world wide. ${ }^{78}$ The enzyme plays an important role in antioxidant mechanisms. In some instances, overwhelming haemolysis akin to favism may result in hyperbilirubinaemia. ${ }^{9}$ However, in many G6PD deficient neonates, only moderately raised haemolysis has been detected by $\mathrm{COHbc}$ techniques, and no correlation found between COHbc and STB. ${ }^{10}$ In contrast, there appears to be a predilection for diminished bilirubin conjugation in the mechanism of jaundice in the G6PD deficient state. ${ }^{11-13}$

With regard to borderline prematurity, ${ }^{14}$ activity of the bilirubin conjugating enzyme, UDP-glucuronosyltransferase lAl (UGT), has been shown to increase in concert with gestational age. ${ }^{15}$ Incomplete maturation of this enzyme in premature infants may therefore result in diminished bilirubin conjugation, placing these neonates at especial risk of hyperbilirubinaemia.

We quantified $\mathrm{COHbc}$ and unconjugated and conjugated serum bilirubin fractions in blood and serum sampled simultaneously from G6PD deficient neonates, to enable

Abbreviations: $\mathrm{COH} b$, carboxyhaemoglobin; $\mathrm{COHbc}, \mathrm{COHb}$ corrected for inspired (room air) CO; G6PD, glucose-6-phosphate dehydrogenase; HPLC, high performance liquid chromatography; STB, serum total bilirubin; TCB, total conjugated bilirubin tion along with efficient bilirubin conjugation can be expected to result in low $\mathrm{COHbc}$ and high TCB and therefore 

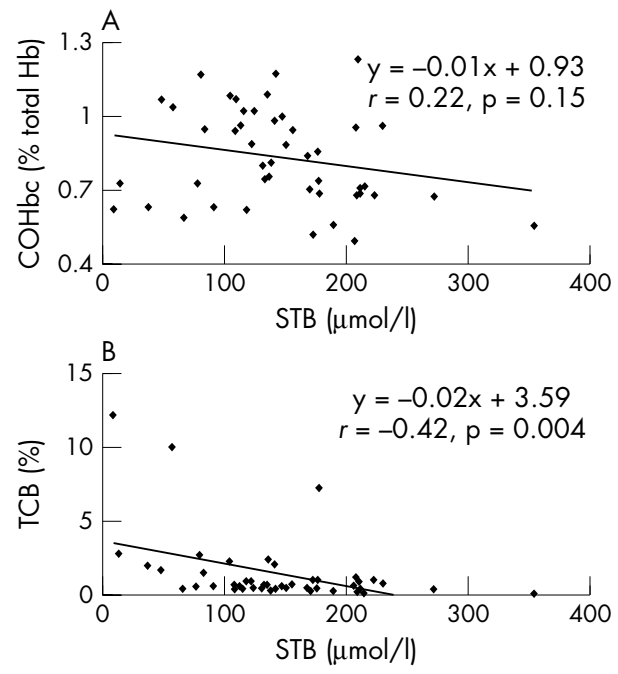

Figure 1 (A) Regression analysis between blood carboxyhaemoglobin corrected for inspired (room air) carbon monoxide ( $\mathrm{COHbc}$ ) and serum total bilirubin (STB). (B) Regression analysis between total conjugated bilirubin (TCB) and STB. Hb, Haemoglobin.

concurrent comparison of bilirubin production and its conjugation. The combined effect of these processes was evaluated using the production-conjugation index, and the contribution of these variables to STB was assessed. The subgroup of neonates of 35-37 weeks gestation was specifically analysed to determine any effect of borderline prematurity on bilirubin production, bilirubin conjugation, and the index.

\section{METHODS}

The study protocol, approved by the institutional review board of the Shaare Zedek Medical Center, was identical with that used previously for G6PD normal neonates of this cohort. $^{56}$ The study cohort comprised healthy consecutive neonates delivered at or near term at the Shaare Zedek Medical Center all of whom were tested for G6PD deficiency. In this study, we analysed those found to be G6PD deficient. Only male infants were included, to avoid the problem of female G6PD deficient heterozygotes for whom accurate designation of G6PD status may be difficult. ${ }^{7}$ Infants with conditions likely to increase the incidence of hyperbilirubinaemia, such as a direct Coombs' positive haemolytic setting, sepsis, maternal diabetes, extensive bruising, and Down's syndrome, were excluded. All babies received routine clinical management during their nursery stay, and clinical management of jaundice was performed using total plasma bilirubin measurements obtained by the diazo method.

A sample of blood was drawn near the beginning of the third day of life. This time was chosen to coincide with routine metabolic screening, avoiding the need to take blood expressly for this study. The study blood sample was divided into aliquots: whole blood for COHb determination ( $150 \mu \mathrm{l}$ ) was collected into custom made capillary tubes containing heparin and saponin, as previously described ${ }^{1617}$ and stored at $-18^{\circ} \mathrm{C}$ until the thawed samples were transported on ice to Stanford University; $0.3-0.5 \mathrm{ml}$ whole blood was collected into an EDTA containing tube for extraction of DNA; $0.5 \mathrm{ml}$ serum was separated, stored in the dark at $-70^{\circ} \mathrm{C}$, and subsequently shipped on dry ice to the University of Padua, Italy, for analysis of the unconjugated and conjugated bilirubin fractions. Concurrent with the blood collection, a sample of room air from the nursery in which the baby was being cared, representing inspired air, was collected and

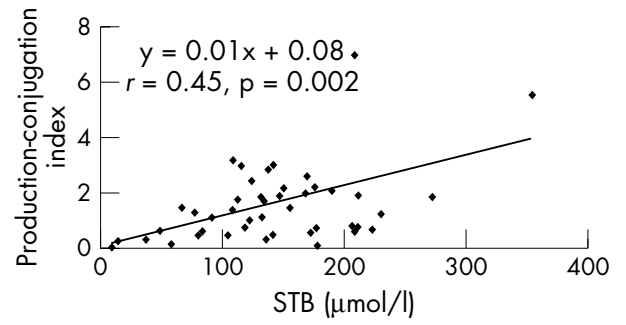

Figure 2 Regression analysis between production-conjugation index and serum total bilirubin (STB).

stored in a special container designed for this purpose pending determination of its CO concentration (Bistable Gas Sampler; Chemical Projects Limited, Toronto, Ontario, Canada).

$\mathrm{COHb}$ was determined by a sensitive and accurate gas chromatographic method, and its concentrations expressed as a percentage of total haemoglobin, which was quantified by the cyanmethaemoglobin method, both as previously described. ${ }^{16}{ }^{17}$ The within day and between day coefficients of variation for reference blood samples with this method are $3 \%$ and $8 \%$ respectively. ${ }^{18}$ The CO concentration of the specimens of room air was determined at the Shaare Zedek Medical Center using a sensitive electrochemical CO analyser supplied by Stanford University. ${ }^{19}$ These CO concentrations were used to correct measured $\mathrm{COHb}$ for the effect of inspired $\mathrm{CO}(\mathrm{COHbc})$ by a previously derived formula $(\mathrm{COHbc}=$ measured $\mathrm{COHb}-0.17 \mu \mathrm{lCO} /$ litre room air). ${ }^{20}$

Unconjugated and conjugated bilirubin fractions were quantified using alkaline methanolysis followed by reverse phase high performance liquid chromatography (HPLC), by the method of Muraca and Blanckaert. ${ }^{21}$ For this method, the within day coefficient of variation is $5-8 \%$, and the between day coefficient of variation is $6-13 \%$. The sum of the measured concentrations of serum unconjugated, monoconjugated, and diconjugated bilirubin was defined as serum total bilirubin (STB), and that of the monoconjugated and diconjugated bilirubin concentrations comprised the total conjugated bilirubin (TCB) value. The latter was expressed as a percentage of the STB concentration, as explained elsewhere. ${ }^{6}$

DNA was prepared from peripheral leucocytes by a high salt extraction method. ${ }^{22}$

The G6PD Mediterranean C563T mutation, which is encountered in Sephardic Jews, ${ }^{13}{ }^{23}$ was determined at The Scripps Research Institute, La Jolla, California, USA (by Ernest Beutler) by polymerase chain reaction followed by allele specific oligonucleotide hybridisation, as described elsewhere. $^{13}$

The relative roles of increased haem catabolism and diminished bilirubin conjugation in the pathogenesis of neonatal bilirubinaemia were compared by correlating $\mathrm{COHbc}$ and TCB respectively with STB, using multivariate analysis. The effects of these processes in combination were evaluated by calculating the bilirubin production-conjugation index, $\mathrm{COHbc/TCB}$, and testing these values for correlation with STB using linear regression analysis. To confirm these findings, the value for the 90th centile for $\mathrm{COHbc}$, TCB, and the production-conjugation index were determined. STB of neonates with values of each of the three variables $\geqslant 90$ th centile were compared with those <90th centile using Student's $t$ test. The effect of borderline prematurity on the production-conjugation index and its constituents was studied by comparing values for neonates $\leqslant 37$ weeks gestational age with those $>37$ weeks, using Student's $t$ test or the Mann-Whitney rank sum test, as appropriate, for 
Table 1 Comparison of STB concentrations for respective $\mathrm{COHbc}$, TCB, and production conjugation index values $\geqslant 90$ th centile versus $<90$ th centile

\begin{tabular}{llll}
\hline \multirow{2}{*}{ Variable } & \multicolumn{2}{l}{ STB concentration $(\mu \mathrm{mol} / \mathrm{I})$} & \\
\cline { 2 - 3 } & $\geqslant 90$ th centile & $<90$ th centile & p Value \\
\hline COHbc (90th centile 1.09) & $134(49)$ & $146(70)$ & 0.7 \\
TCB (90th centile 2.65) & $67(68)$ & $154(62)$ & 0.005 \\
Production-conjugation index (90th centile 3.0) & $205(94)$ & $137(61)$ & 0.03 \\
\hline
\end{tabular}

Values are mean (SD).

STB, Serum total bilirubin; $\mathrm{COHbc}$, carboxyhaemoglobin corrected for inspired carbon monoxide; TCB, total conjugated bilirubin.

Table 2 Comparison of values for the production-conjugation index, $\mathrm{COHbc}$, TCB, and STB for neonates $\geqslant 37$ weeks gestation and those $>37$ weeks

\begin{tabular}{llll}
\hline Variable & $\leqslant \mathbf{3 7}$ weeks gestation & $\mathbf{3 7}$ weeks gestation & $\mathbf{p}$ Value \\
\hline $\begin{array}{l}\text { Production-conjugation } \\
\text { index (unitless) }\end{array}$ & $2.31(2.12-3.08)$ & $1.05(0.53-1.81)$ & 0.003 \\
COHbc (\% Hb) & $0.88(0.21)$ & $0.82(0.20)$ & 0.46 \\
TCB (\% STB) & $0.39(0.31-0.42)$ & $0.74(0.44-1.69)$ & 0.009 \\
STB $(\mu \mathrm{mol} / \mathrm{l})$ & $160(35)$ & $141(72)$ & 0.48 \\
\hline
\end{tabular}

Values are mean (SD) or median (interquartile range), as appropriate

STB, Serum total bilirubin; $\mathrm{COHbc}$, carboxyhaemoglobin corrected for inspired carbon monoxide; TCB, total conjugated bilirubin; $\mathrm{H} \mathrm{Hb}$, total haemoglobin.

values with a parametric or non-parametric distribution respectively. Parametric data are presented as mean (SD), and non-parametric data as median (interquartile range). Significance is defined as $\mathrm{p}<0.05$.

\section{RESULTS}

A total of 51 G6PD deficient neonates was sampled at 51 (8) hours of age. Gestational age was 39 (1) weeks, and birth weight was 3118 (462) g. Eight were born at $\geqslant 37$ weeks gestation. Ten $(20 \%)$ were delivered by caesarean section, 26 $(51 \%)$ were exclusively breast fed, and a further nine $(18 \%)$ received a combination of breast feeding and formula. Sixteen $(31 \%)$ developed hyperbilirubinaemia, defined as a plasma total bilirubin $\geqslant 256 \mu \mathrm{mol} / \mathrm{l}(15.0 \mathrm{mg} / \mathrm{dl})$.

Measured values for the entire G6PD deficient neonatal population included mean (HPLC) STB concentration 144 (67) $\mu \mathrm{mol} /$, COHbc level $0.83(0.19) \%$, and median (interquartile range) TCB value $0.65 \%(0.39-1.27 \%)$. Multivariate analysis with STB as the dependent variable and COHbc and TCB as independent variables showed no correlation with COHbc, but did show correlation with TCB $(r=0.50, \mathrm{p}=$ 0.09 , and $\mathrm{p}=0.003$ for $\mathrm{COHbc}$ and TCB respectively). Figure 1 shows the correlation analysis between STB, and COHbc and TCB. The median production-conjugation index was $1.25(0.63-2.07)$. The index correlated positively with STB $(r=0.45, \mathrm{p}=0.002$; fig 2$)$. Table 1 gives values for the 90th centile for $\mathrm{COHbc}$, TCB, and the production-conjugation index along with STB values for each variable $\geqslant 90$ th and $<90$ th centile. STB concentrations were not significantly higher in those with $\mathrm{COHbc} \geqslant 90$ th centile than those $<90$ th centile, but were significantly lower in those with TCB values $\geqslant 90$ th centile than those $<90$ th centile. Similarly, STB concentrations were higher in those with productionconjugation index values $\geqslant 90$ th centile than those with lower index values.

Table 2 summarises values for neonates born $\leqslant 37$ weeks compared with those born $>37$ weeks. The productionconjugation index was significantly higher in the near term group than in the more mature neonates. Whereas COHbc was not significantly affected by borderline prematurity, TCB was significantly lower in this group. Although STB concentrations were not significantly different between the two groups, there was a trend to a weak correlation between diminishing gestational age and increasing STB concentration $(r=0.28, \mathrm{p}=0.06)$.

\section{DISCUSSION}

This study differs from previous ones of this nature ${ }^{10-13}$ in that COHbc and TCB were analysed in blood and serum sampled simultaneously from the same patients, allowing direct comparison between the bilirubin production and conjugative processes at the same point in time, and thereby providing insight into the physiological relations between production and conjugation in these infants. Furthermore, whereas in previous studies $\mathrm{COHbc}$ in G6PD deficient neonates was analysed in relation to STB concentrations determined by the diazo method, we used a highly accurate reverse phase HPLC method, which allowed determination of both unconjugated and conjugated bilirubin fractions.

The G6PD deficient neonates of this study differ from the previously reported G6PD normal counterparts drawn from the same cohort in that STB concentrations were higher, and the risk of hyperbilirubinaemia greater. ${ }^{5}$ For reference, STB concentrations were 144 (67) $\mu \mathrm{mol} / \mathrm{l} v 114(67) \mu \mathrm{mol} / \mathrm{l}(\mathrm{p}=$ $0.003)$, and the incidence of clinically determined hyperbilirubinaemia (plasma bilirubin $\geqslant 256 \mu \mathrm{mol} / \mathrm{l}$ by the diazo method) was $31 \% \vee 6 \%(\mathrm{p}<0.001)$. The study design, by which measurements reflecting haem catabolism and bilirubin conjugation were quantified from simultaneously drawn samples from identical babies, facilitated comparison of the roles of these processes in the pathophysiology of jaundice. As previously shown using serum bilirubin determinations obtained by the diazo method, ${ }^{10}{ }^{24} \mathrm{COHbc}$ did not correlate with HPLC determined STB concentrations in the G6PD deficient neonates. Correspondingly, $\mathrm{COHbc}$ values $\geqslant 90$ th centile were not associated with STB concentrations greater than those with $\mathrm{COHbc}$ values <90th centile, as would be expected in a G6PD normal population. In contrast, TCB did diminish progressively along with increasing STB concentration, whereas STB concentrations in neonates with TCB bilirubin concentrations $\geqslant 90$ th centile, representing those with the most efficient bilirubin conjugation, were 


\section{What is known on this topic}

G6PD deficiency is associated with increased haemolysis, as shown by $\mathrm{COHb}$ studies. However, in contrast with G6PD normal neonates, in whom $\mathrm{COHb}$ can be expected to correlate with increasing STB, $\mathrm{COHb}$ does not correlate with STB in the G6PD deficient state. Studies of serum conjugated bilirubin fractions, an index of bilirubin conjugation, have shown a propensity to diminished bilirubin conjugation in G6PD deficient neonates compared with that of the normal population.

lower than those with higher TCB concentration. These findings confirm a tendency for inefficient bilirubin conjugation over and above increased haemolysis, in the pathogenesis of bilirubinaemia in G6PD deficient neonates. ${ }^{11-13}$ The production-conjugation index, reflecting imbalance between bilirubin production and its conjugation, increased in concert with increasing STB concentration, confirming the concept of imbalance between production and conjugation of bilirubin in the pathogenesis of jaundice in a neonatal subgroup with a higher than average incidence of hyperbilirubinaemia. Even when possible outliers with the two highest indices were removed, the correlation between STB and the index maintained significance (fig 2).

Borderline prematurity is an important contributing factor to hyperbilirubinaemia and kernicterus. ${ }^{14}$ The high risk status of neonates of 35-37 weeks gestation has been emphasised by the American Academy of Pediatrics Subcommittee on Neonatal Hyperbilirubinaemia. ${ }^{25}$ Furthermore, in a recent report of 61 neonates, originally discharged as healthy, but readmitted for kernicterus within the first seven days, $23 \%$ were of 35-36 weeks gestational age. ${ }^{26}$ In our study, although there was only a trend to a weak correlation between diminishing gestational age and STB concentration, production-conjugation indices, in contrast, were significantly higher in the near term subgroup. Further analysis pinpointed this difference to the TCB component of the index, implying diminished bilirubin conjugation, but not increased haem catabolism, in the near term neonates. Although the effect of borderline prematurity on STB concentration was minimal, that on the production-conjugation index was highly significant, and may contribute to our understanding of the high risk of near term neonates. The degree of imbalance between bilirubin production and conjugation at the time the neonates were sampled was insufficient to result in significantly higher STB concentrations. However, in a situation with either diminished bilirubin conjugation or increased haem catabolism, however slight, any additional or superimposed icterogenic factors may have a much greater effect, with the potential of severe hyperbilirubinaemia, than if the bilirubin production and conjugation processes were working at normal capacity.

The COHbc measurements we used were obtained by state of the art technology. Red blood cell indices and haptoglobin or haemopexin determinations are unreliable indicators of haemolysis in the newborn because of overlap between the normal and haemolytic states. As the predominant source of endogenous CO in the human body is from the degradation of haem by haem oxygenase, with little originating from nonhaem sources, measurement of blood COHbc reflects primarily the rate of bilirubin formation from haemoglobin. ${ }^{121617}$ The serum conjugated bilirubin is believed to reflect the intrahepatocytic bilirubin profile, as the liver is the only organ that can esterify bilirubin to any significant extent, and conjugated bilirubin compounds are not absorbed from the bowel. In the absence of hepatocellular disease or

\section{What this study adds}

Whereas $\mathrm{COHb}$ and serum conjugated bilirubin assays were previously performed in different neonatal populations, in this study the haemolytic and conjugative processes were studied in the same neonates at the same point in time. Thus these processes can be directly compared and contrasted. STB was determined using a highly accurate reverse phase HPLC method capable of measuring both unconjugated and conjugated bilirubin fractions, rather than just the total. The relations between bilirubin production and its conjugation are evaluated in G6PD deficient neonates, and the concept of imbalance between these processes in the pathogenesis of jaundice is substantiated. In addition, a subset of borderline preterm neonates $\leqslant 37$ weeks gestation is compared and contrasted with term neonates.

cholestasis, this method provides a minimally invasive assessment of bilirubin conjugation. ${ }^{3}{ }^{4}$ The assay has been used for this purpose in neonates, children, and adults. ${ }^{6}$ In individual cases, variation in the balance between the rate limiting steps and mild disparities in bile flow may affect interpretation of the results; however, use of large numbers of subjects should offset this potential inaccuracy. The pathogenesis of neonatal jaundice is clearly multifactorial. ${ }^{14}$ COHbc and TCB studies evaluate some, but not all, of the contributing factors. Some of the latter include hepatic uptake, bilirubin excretion, and reabsorption of bilirubin via the enterohepatic circulation.

Arithmetic deficiencies of this index or its components include the facts that $\mathrm{COHb}$ is measured as a percentage of total $\mathrm{Hb}$, but that catabolism of haem is what the COHbc is expected to reflect. TCB is expressed as a percentage of the STB, but it is the STB that is subsequently correlated with the production-conjugation index. Notwithstanding these mathematical dependencies and the already mentioned difficulties in interpretation, the index does contribute to our understanding of the mechanism of neonatal hyperbilirubinaemia. An increase in the index in line with rising STB concentration confirms the importance of the concept that imbalance between bilirubin production and its conjugation is a crucial factor in the mechanism of neonatal jaundice. Diminishing gestational age has a further effect on increasing the degree of imbalance. Additional genetic or environmental factors that either increase haemolysis or diminish bilirubin conjugation may exacerbate any imbalance between these processes, potentiating the risk of severe neonatal hyperbilirubinaemia.

\section{ACKNOWLEDGEMENTS}

We are grateful to Ernest Beutler, MD, of The Scripps Research Institute, La Jolla, California, USA, for G6PD genotyping. We thank Mr Ronald J Wong for technical and editorial assistance. The study was supported at Shaare Zedek Medical Center by grants from the Golden Charitable Trust, London, UK and the Mirsky Research Fund, and at Stanford University by National Institutes of Health grant RR00070, the Hess Research Fund, the HM Lui Research Fund, and the Mary L Johnson Research Fund.

\section{Authors' affiliations}

M Kaplan, C Hammerman, Department of Neonatology, Shaare Zedek Medical Center, Jerusalem, Israel

M Kaplan, Faculty of Medicine, Hebrew University, Jerusalem, Israel C Hammerman, Faculty of Health Sciences, Ben Gurion University of the Negev, Be'er Sheva, Israel

M Muraca, M T Vilei, Department of Internal Medicine, University of Padua, Florence, Italy 
F F Rubaltelli, Department of Neonatology, University of Florence, Florence, Italy

H J Vreman, D K Stevenson, Division of Neonatal and Developmental Medicine, Department of Pediatrics, Stanford University Medical Center, Stanford, CA, USA

*Present address: Clinical Chemistry Laboratory, Ospedale Pediatrico Bambino Gesu, Piazza Sant' Onofrio 4, 00165 Rome, Italy

Competing interests: none declared

Presented in part at the Pediatric Academic Societies' Annual Meeting, Seattle, WA, USA, 3-6 May, 2003.

\section{REFERENCES}

1 Stevenson DK, Dennery PA, Hintz SR. Understanding neonatal jaundice. J Perinatol 2001;21:S21-4.

2 Vreman HJ, Mahoney JJ, Stevenson DK. Carbon monoxide and carboxyhemoglobin. Adv Pediatr 1995;42:303-34.

3 Muraca M, Fevery J, Blanckaert N. Relationships between serum bilirubins and production and conjugation of bilirubin. Studies in Gilbert's syndrome, Crigler-Naijar disease, hemolytic disorders, and rat models. Gastroenterology 1987; $92: 309-17$

4 Berg Cl, Crawford JM, Gollan JL. Bilirubin metabolism and the pathophysiology of jaundice. In: Schiff ER, Sorrel MF, Maddrey WC, eds. Shiff's diseases of the liver. Philadelphia: Lippincott-Raven, 1999:147-92.

5 Kaplan M, Muraca M, Hammerman C, et al. Imbalance between production and conjugation of bilirubin: a fundamental concept in the mechanism of neonatal jaundice. Pediatrics 2002;110(4). www.pediatrics.org/cgi/content/ full/110/4/e47.

6 Kaplan M, Hammerman C, Rubaltelli FF, et al. Hemolysis and bilirubin conjugation in association with UDP-glucuronosyltransferase $1 \mathrm{Al}$ promoter polymorphism. Hepatology 2002;35:905-11.

7 Beutler E. G6PD deficiency. Blood 1994;84:3613-36.

8 Kaplan M, Hammerman C. Glucose-6-phosphate dehydrogenase deficiency: a potential source of severe neonatal hyperbilirubinaemia and kernicterus. Semin Neonatol 2002;7:121-8.

9 Slusher TM, Vreman, HJ, McLaren DW, et al. Glucose-6-phosphate dehydrogenase deficiency and carboxyhemoglobin concentrations associated with bilirubin-related morbidity and death in Nigerian infants. J Pediatr 1995; 126:102-8.

10 Kaplan M, Vreman HJ, Hammerman C, et al. Contribution of haemolysis to jaundice in Sephardic Jewish glucose-6-phosphate dehydrogenase deficient neonates. Br J Haematol 1996;93:822-7.

11 Kaplan M, Rubaltelli FF, Hammerman C. Conjugated bilirubin in neonates with glucose-6-phosphate dehydrogenase deficiency. J Pediatr 1996; 128:695-7.
12 Kaplan M, Muraca M, Hammerman C, et al. Bilirubin conjugation, reflected by conjugated bilirubin fractions, in glucose-6-phosphate dehydrogenasedeficient neonates: a determining factor in the pathogenesis of hyperbilirubinemia. Pediatrics 1998;102(3). www. pediatrics.org/cgi/ content/full/102/3/e37.

13 Kaplan M, Renbaum P, Levy-Lahad E, et al. Gilbert's syndrome and glucose6-phosphate dehydrogenase deficiency: a dose dependent genetic interaction crucial to neonatal hyperbilirubinemia. Proc Natl Acad Sci USA 1997;94:12128-32.

14 Dennery PA, Seidman DS, Stevenson DK. Neonatal hyperbilirubinemia. N Engl J Med 2001;344:581-90.

15 Kawade N, Onishi S. The prenatal and postnatal development of UDP-glucuronyltransferase activity towards bilirubin and the effect of premature birth on this activity in the human liver. Biochem $J$ $1981 ; 196: 257-60$.

16 Vreman HJ, Kwong LK, Stevenson DK. Carbon monoxide in blood: an improved microliter blood-sample collection system, with rapid analysis by gas chromatography. Clin Chem 1984;30:1382-5.

17 Vreman HJ, Stevenson DK, Zwart A. Analysis for carboxyhemoglobin by gas chromatography and multicomponent spectrophotometry compared. Clin Chem 1987;33:694-7.

18 Widness JA, Lowe LS, Stevenson DK, et al. Direct relationship of fetal carboxyhemoglobin with hemolysis in alloimmunized pregnancies. Pediatr Res 1994:35:713-19.

19 Vreman HJ, Stevenson DK, Oh W, et al. Semiportable electrochemical instrument for determining carbon monoxide in breath. Clin Chem 1994:40:1927-33

20 Ostrander CR, Cohen RS, Hopper AO, et al. Paired determinations of blood carboxyhemoglobin concentrations and carbon monoxide excretion rate in term and preterm infants. J Lab Clin Med 1982;100:745-55.

21 Muraca M, Blanckaert N. Liquid-chromatographic assay and identification of mono- and diester conjugates of bilirubin in normal serum. Clin Chem 1983;29:1767-71.

22 Miller SA, Dykes DD. Polesky HF. A simple salting out procedure for extracting DNA from human nucleated cells. Nucleic Acids Res 1988;16:1215.

23 Oppenheim A, Jury CL, Rund D, et al. G6PD Mediterranean accounts for the high prevalence of G6PD deficiency in Kurdish Jews. Hum Genet 1993;91:293-4.

24 Kaplan M, Hammerman C, Renbaum P, et al. Differing pathogenesis of perinatal bilirubinemia in glucose-6-phosphate dehydrogenase-deficient versus normal neonates. Pediatr Res 2001;50:532-7.

25 American Academy of Pediatrics, Subcommittee on Neonatal Hyperbilirubinemia. Neonatal jaundice and kernicterus. Pediatrics $2001 ; 108: 763-5$

26 Johnson LH, Bhutani VK, Brown AK. System-based approach to management of neonatal jaundice and prevention of kernicterus. I Pediatr $2002 ; 140: 396-403$. 